\begin{tabular}{|c|l|}
\hline Title & $\begin{array}{l}\text { Electronic transmission in the lateral heterostructure of semiconducting and metall lic transition-metal dichal cogenide } \\
\text { monolayers }\end{array}$ \\
\hline Author(s) & Habe, Tetsuro \\
\hline Citation & $\begin{array}{l}\text { Journal of A pplied Physics, 126(12), 123901 } \\
\text { https://doi.org/10.1063/1.5098802 }\end{array}$ \\
\hline Issue Date & 2019-09-28 \\
\hline Doc URL & http://hdl.handle.net/2115/19315 \\
\hline Rights & $\begin{array}{l}\text { The following article appeared in Journal of A pplied Physics 126, 123901 (2019) and may be found at } \\
\text { https://doi.org/10.1063/1.5098802. }\end{array}$ \\
\hline Type & article \\
\hline File Information & \begin{tabular}{l}
$1.5098802 . p d f$ \\
\hline
\end{tabular} \\
\hline
\end{tabular}

Instructions for use 


\section{Electronic transmission in the lateral heterostructure of semiconducting and metallic transition-metal dichalcogenide monolayers}

Cite as: J. Appl. Phys. 126, 123901 (2019); https://doi.org/10.1063/1.5098802

Submitted: 03 April 2019. Accepted: 05 September 2019. Published Online: 23 September 2019

\section{Tetsuro Habe}

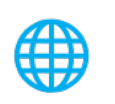

View Online

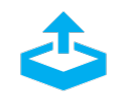

Export Citation

\section{ARTICLES YOU MAY BE INTERESTED IN}

Conduction mechanisms in ZnO nanowires based Schottky diode grown under an electric field

Journal of Applied Physics 126, 124501 (2019); https://doi.org/10.1063/1.5117171

Impact of defects on the electrical properties of $\mathrm{p}-\mathrm{n}$ diodes formed by implanting $\mathrm{Mg}$ and $\mathrm{H}$ ions into N-polar GaN

Journal of Applied Physics 126, 125102 (2019); https://doi.org/10.1063/1.5116886

Defect energy levels in carbon implanted n-type homoepitaxial GaN

Journal of Applied Physics 126, 125301 (2019); https://doi.org/10.1063/1.5109237

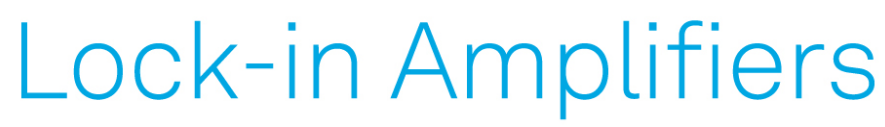

... and more, from DC to $600 \mathrm{MHz}$ Watch 


\title{
Electronic transmission in the lateral heterostructure of semiconducting and metallic transition-metal dichalcogenide monolayers
}

Cite as: J. Appl. Phys. 126, 123901 (2019); doi: 10.1063/1.5098802

Submitted: 3 April 2019 - Accepted: 5 September 2019.

Published Online: 23 September 2019

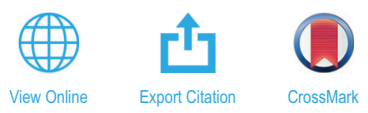

Tetsuro Habe ${ }^{a)}$

AFFILIATIONS

Department of Applied Physics, Hokkaido University, Sapporo, Hokkaido 060-0808, Japan

${ }^{a)}$ Author to whom correspondence should be addressed: tet.habe@gmail.com

\begin{abstract}
We investigate the electronic transport property of lateral heterojunctions of semiconducting and metallic transition-metal dichalcogenide monolayers, $\mathrm{MoSe}_{2}$ and $\mathrm{NbSe}_{2}$, respectively. We calculate the electronic transmission probability by using a multiorbital tight-binding model based on the first-principles band structure. The transmission probability depends on the spin and valley degrees of freedom. This dependence qualitatively changes by the interface structure. The heterostructure with a zigzag interface preserves the spin and the valley of electrons in the transmission process. On the other hand, the armchair interface enables conduction electrons to transmit with changing the valley and increases the conductance in the hole-doped junctions due to the valley-flip transmission. We also discuss the spin and valley polarizations of electronic current in the heterojunctions.
\end{abstract}

Published under license by AIP Publishing. https://doi.org/10.1063/1.5098802

\section{INTRODUCTION}

Transition-metal dichalcogenides (TMDCs) are layered materials of atomically thin two-dimensional crystals consisting of transitionmetal and chalcogen atoms, and the monolayer has attracted much attention in condensed matter physics. In semiconducting TMDC monolayers, electrons have two discrete degrees of freedom, spin and valley, due to the band structure. ${ }^{1,2}$ Strong spin-orbit coupling (SOC) leads to spin-related physics in TMDCs, ${ }^{3,4}$ and the valley-dependent Berry curvature causes the valley Hall effect. ${ }^{5}$ Therefore, TMDC monolayers have been researched for applications in spintronics ${ }^{6-9}$ and valleytronics. ${ }^{10-13}$ Since the electronic spin direction is locked to the valley due to the strong SOC, the valleytronics and the spintronics can be combined, e.g., the valley can be detected as the spin.

Stable monolayers can be fabricated by cleaving from a crystal $^{14-16}$ or chemical vapor deposition (CVD) on a substrate. ${ }^{17,18}$ The experimental techniques enable composing heterostructures consisting of different atomic layers. The first-designed heterostructure is a stacking of different atomic layers, the so-called van der Waals heterostructure, and it has opened several areas of research and application: optics, ${ }^{19}$ electric transport, ${ }^{20}$ and exciton physics. ${ }^{21,22}$ The lateral heterostructure is composed of two atomic layers bonded as a single layer and that of semiconducting TMDCs has been realized by CVD. ${ }^{23-2}$
The semiconducting heterojunction has been investigated theoretically ${ }^{8,30,31}$ and experimentally applied to the p-n junction, ${ }^{32,33}$ valleytronics, ${ }^{34}$ and optoelectronics. ${ }^{35}$ This junction provides novel electronic properties for semiconducting TMDCs.

Metallic TMDCs have also attracted much attention in terms of condensed phases. The TMDC of the group- $\mathrm{V}$ transition-metal atoms, $\mathrm{Nb}$ and $\mathrm{Ta}$, is metallic and has been discovered to show superconductivity $^{36,37}$ and the charge density wave (CDW), ${ }^{38,39}$ even in the monolayer. The $\mathrm{NbSe}_{2}$ monolayer, even in the normal phase, has qualitatively different properties of conduction electrons from the semiconducting monolayers. There are three Fermi pockets in the first Brillouin zone, whereas the semiconducting members have two pockets called the $K$ and $K^{\prime}$ valleys. Each pocket has both the up-spin and down-spin states. Thus, the correlation between the spin and valley degrees of freedom, the key property of semiconducting monolayers for combining the spintronics and the valleytronics, is absent in the metallic monolayers. We consider the lateral heterojunction of the metallic and semiconducting TMDC monolayers and show that it induces the correlation between the spin and the three valleys.

In this paper, we report our investigation of the spin- and valleydependent electronic transport property of the lateral heterojunction 
of $\mathrm{MoSe}_{2}$ and $\mathrm{NbSe}_{2}$ monolayers, metallic and semiconducting TMDCs, respectively. We consider two types of interface for such a junction as shown in Fig. 1. The electronic transmission is qualitatively different in two types of interface, the armchair interface in (a) and the zigzag interface in (b). We compute the spin and valley dependence of electronic conductance by using the first-principles band calculation and lattice Green's function method, and we show the valley-spin correlated transmission effect. This result gives a fundamental knowledge for spintronics and valleytronics in lateral heterojunctions.

\section{BAND STRUCTURE}

The TMDC monolayer consists of three sublayers, a sublayer of transition-metal atoms sandwiched by two sublayers of

(a)

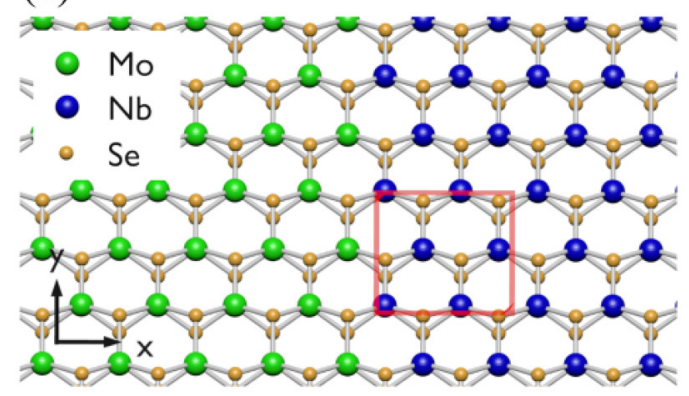

(b)
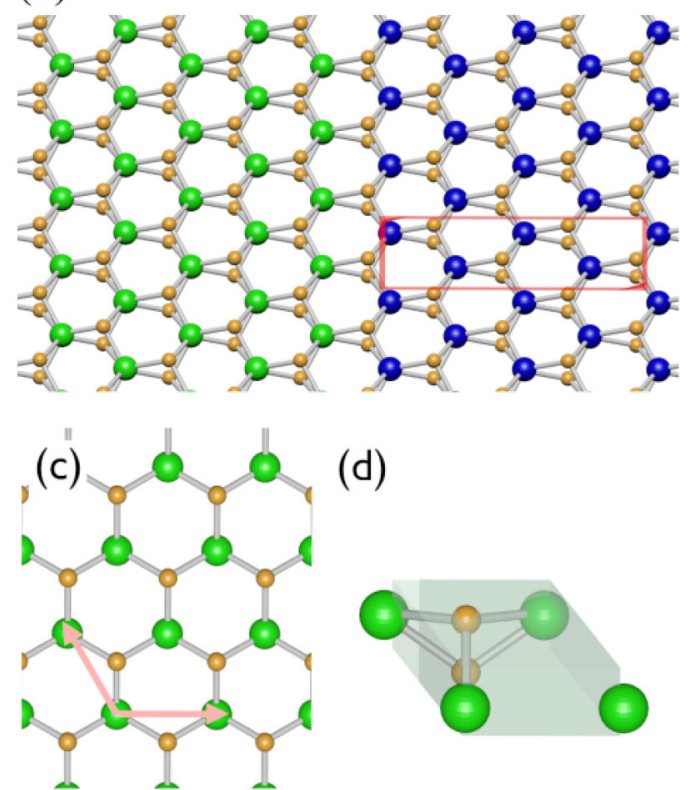

(d)

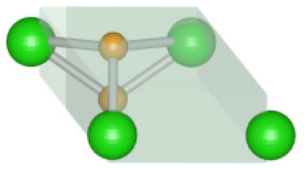

FIG. 1. The schematics of the atomic heterostructure of $\mathrm{MoSe}_{2}$ and $\mathrm{NbSe}_{2}$ with an armchair interface (a) and a zigzag interface (b). The unit cell of this junction is indicated by a square frame in (a) and (b). The crystal structure and the primitive unit cell of TMDC monolayer in (c) and (d), respectively. The two arrows indicate the lattice vectors. chalcogen atoms. The transition-metal atoms, $\mathrm{Nb}$ or Mo, and chalcogen atoms, Se, are strongly bonded and form a two-dimensional hexagonal lattice as shown in Fig. 1(c).

We plot the band structure of the pristine monolayer of $\mathrm{NbSe}_{2}$ and $\mathrm{MoSe}_{2}$ in Figs. 2(a) and 2(b), respectively. These bands are calculated by using quantum-ESPRESSO, a first-principles calculation code. ${ }^{40}$ Here, we adopt a projector augmented wave (PAW) method with a generalized gradient approximation (GGA) functional including SOC, the cut-off energy of the plane wave basis $50 \mathrm{Ry}$, and the conversion criterion $10^{-8}$ Ry. The lattice parameters are also calculated by the lattice-relaxation code of quantum-ESPRESSO as $a_{\mathrm{Nb}-\mathrm{Nb}}=3.476 \AA$ and $d_{\mathrm{Se}-\mathrm{Se}}=3.514 \AA$ in $\mathrm{NbSe}_{2}$ and $a_{\mathrm{Mo}-\mathrm{Mo}}=3.319 \AA$ and $d_{\mathrm{Se}-\mathrm{Se}}=3.343 \AA$ in $\mathrm{MoSe}_{2}$, where $a_{M-M}\left(d_{\mathrm{X}-\mathrm{X}}\right)$ is the horizontal (vertical) distance between nearest neighbor transition-metal (chalcogen) atoms.

The $\mathrm{MoSe}_{2}$ monolayer is a semiconductor and has a direct gap at the $K$ and $K^{\prime}$ points in the Brillouin zone. At the band edge, the electronic states split into two spin states due to SOC (see Fig. 2), where the spin-split in the valence band is much larger than that in the conduction band. Here, the spin axis is restricted in the out-of-plane direction due to the crystal symmetry. ${ }^{1}$ We show the Fermi surface of electron-doped and hole-doped monolayers with the charge density $n=-3 \times 10^{13} \mathrm{~cm}^{-2}$ and $3 \times 10^{13} \mathrm{~cm}^{-2}$,
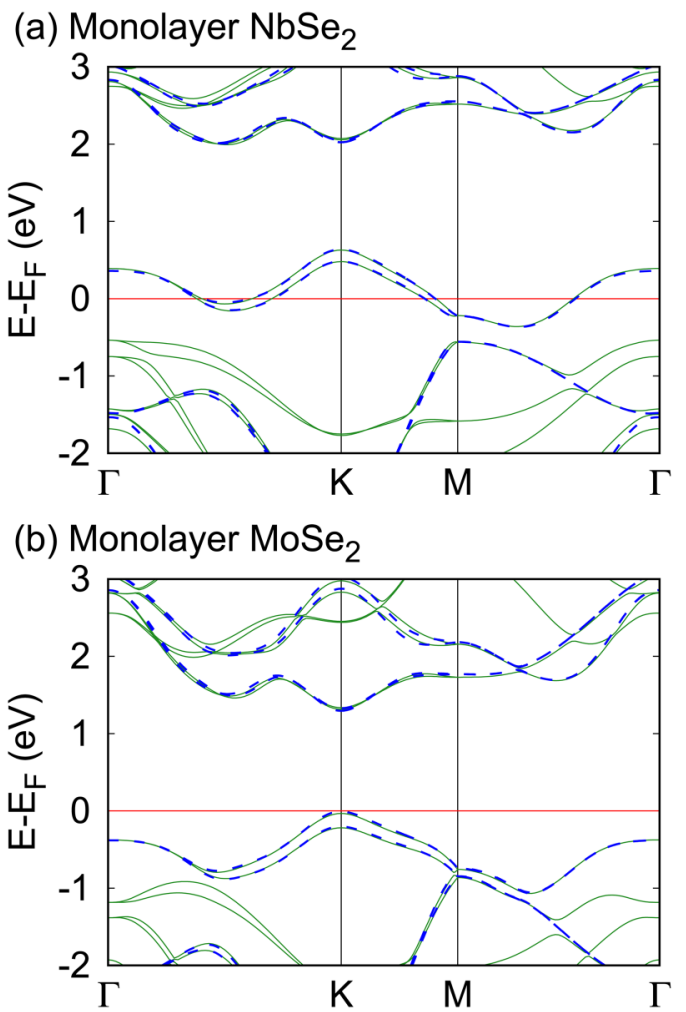

FIG. 2. Band structure of monolayer $\mathrm{NbSe}_{2}$ in (a) and $\mathrm{MoSe}_{2}$ in (b). The horizontal line implies the Fermi energy. The dashed lines indicate the bands computed by a multiorbital tight-binding model (see Sec. III). 
respectively, in Fig. 3. Here, the Fermi energy $E_{F}$ is computed under the condition of a charge density $n$ as

$$
n=n_{0}-\int \frac{d^{2} \boldsymbol{k}}{(2 \pi)^{2}} \theta\left(E_{\alpha, \boldsymbol{k}}-E_{F}\right),
$$

where $n_{0}$ is the charge density of nuclei and $E_{\alpha, k}$ is the energy dispersion in Fig. 2. The Fermi pockets appear around the $K$ and $K^{\prime}$ points, and each pocket is so-called a valley. In the hole-doped monolayer, the spin is fully polarized in each valley up to $|n| \sim 6 \times 10^{13} \mathrm{~cm}^{-2}$, the experimentally feasible charge density by using electrostatic gating. ${ }^{41}$ The spin polarization direction is opposite in two valleys due to the time-reversal symmetry, and total spinpolarization is absent. Under the condition of $n<0$, on the other hand, both the spin states are present in each valley. The electrons in $\mathrm{MoSe}_{2}$ can be characterized by spin and valley indexes at any charge density.

In the $\mathrm{NbSe}_{2}$ monolayer, the band is quite similar to that of $\mathrm{MoSe}_{2}$ due to the crystal structure as shown in Fig. 2, but the band is partially filled even at the charge neutral point because the atomic number of $\mathrm{Nb}$ is one less than Mo. We show the Fermi surface of the pristine $\mathrm{NbSe}_{2}$ monolayer in Fig. 4. The Fermi pockets appear around three high-symmetry points, the $K, K^{\prime}$, and $\Gamma$ points. We represent the pockets as the $K, K^{\prime}$, and $\Gamma$ valleys in what follows. In every valley, both the two spin states are present. The spin polarization is nonzero and opposite to each other in the $K$ and $K^{\prime}$ valleys, but the populations of two spins are equal to each other in the $\Gamma$ valley. Every Fermi pocket is strongly trigonal warping due to the threefold symmetry of the crystal structure.

\section{CALCULATION METHOD}

To calculate the electronic transmission probability, we adopt a tight-binding model defined on the basis of Wannier

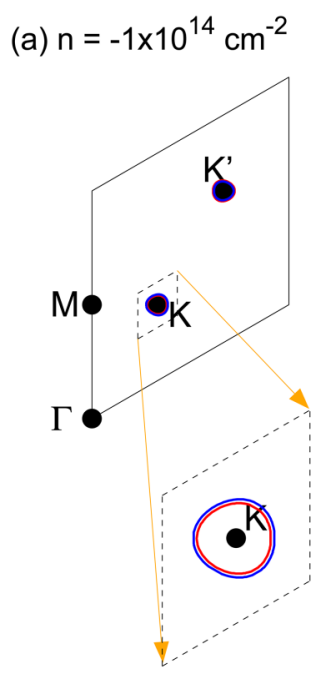

(b) $\mathrm{n}=1 \times 10^{14} \mathrm{~cm}^{-2}$

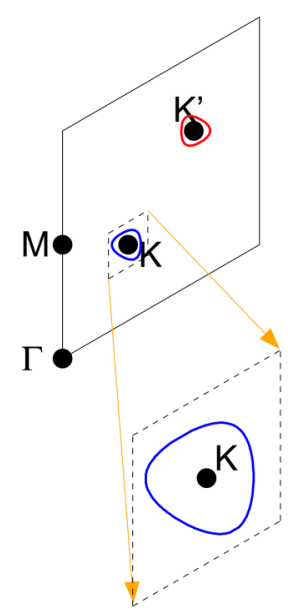

FIG. 3. Fermi surface in (a) electron-doped and (b) hole-doped $\mathrm{MoSe}_{2}$. Up-spin and down-spin states are indicated by blue and red, respectively. (a) Monolayer $\mathrm{NbSe}_{2}$

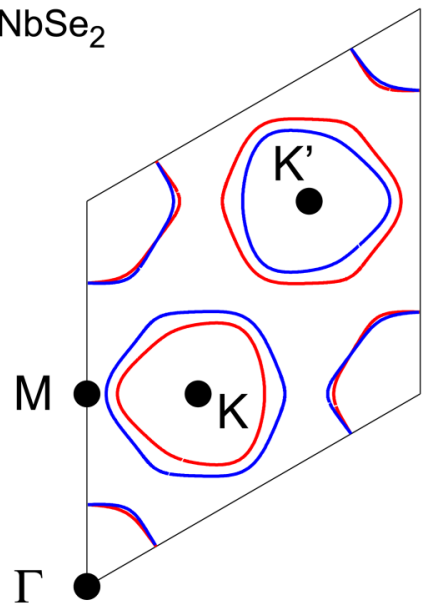

FIG. 4. Fermi surface in pristine monolayer $\mathrm{NbSe}_{2}$.

functions, where the hopping integrals are computed from the first-principles band structure in Sec. II. The maximally localized Wannier functions and spin-dependent hopping integrals are computed by using Wannier90. ${ }^{42}$ Here, we adopt three $d$-orbitals $\left|M, d_{3 z^{2}-r^{2}}\right\rangle,\left|M, d_{x y}\right\rangle$, and $\left|M, d_{x^{2}-y^{2}}\right\rangle$ of the transition-metal atom $M$ and three superpositions of $p$-orbitals of the top selenium $\mathrm{Se}^{t}$ and the bottom one $\mathrm{Se}^{b}$ as $\left|\mathrm{Se}^{+}, p_{x}\right\rangle,\left|\mathrm{Se}^{+}, p_{y}\right\rangle$, and $\left|\mathrm{Se}^{-}, p_{z}\right\rangle$ with $\left|\mathrm{Se}^{ \pm}, p_{v}\right\rangle \equiv\left(\left|\mathrm{Se}^{t}, p_{v}\right\rangle \pm\left|\mathrm{Se}^{b}, p_{v}\right\rangle\right) / \sqrt{2}$. These orbitals have even parity under mirror operation in the $z$ axis, and they are independent of odd parity orbitals in the electronic structure. We show the band structure calculated by the tight-binding model in Fig. 2 . It well reproduces the first-principles bands.

We simulate the electronic transmission in lateral heterostructures by using the multiorbital tight-binding model including the interface between $\mathrm{MoSe}_{2}$ and $\mathrm{NbSe}_{2}$ monolayers. We assume a commensurate interface, where two atomic layers are bonded without dangling and misalignment as shown in Fig. 1 and a periodic boundary condition in the parallel direction to the interface. The incident electronic wave with $k_{y}$ in $\mathrm{MoSe}_{2}$ transmits to $\mathrm{NbSe}_{2}$ as a wave with $k_{y}^{\prime}=\left(a_{\mathrm{Mo}-\mathrm{Mo}} / a_{\mathrm{Nb}-\mathrm{Nb}}\right) k_{y}$, and thus, the transmission coefficient is defined at each $k_{y}$. In what follows, we represent both the wave numbers, $k_{y}$ and $k_{y}^{\prime}$, by $k_{y}$. Despite the mismatch of lattice parameters, such a commensurate heterostructure has been fabricated experimentally. ${ }^{24,32}$ To use the lattice Green's function method, we consider a unit cell including four transition-metal atoms and eight Se as shown in Fig. 1. The choice of the unit cell transforms the tight-binding Hamiltonian into a suitable form for the recursive Green's function method, where it includes only hopping integrals between nearest neighbor cells. Here, we adopt the hopping matrix in $\mathrm{NbSe}_{2}$ as that between $\mathrm{MoSe}_{2}$ and $\mathrm{NbSe}_{2}$ because the outer shell of $\mathrm{Mo}$ and $\mathrm{Nb}$ atoms is the same as $4 d$-orbitals, and the hopping integral is similar to each other in the two monolayers.

We show the charge distribution around the interface of the lateral heterojunction for $n=-3 \times 10^{13} \mathrm{~cm}^{-2}$ and $n=3 \times 10^{13} \mathrm{~cm}^{-2}$ in Fig. 5. They are obtained by using the tight-binding model under 
(a) Zig-zag interface

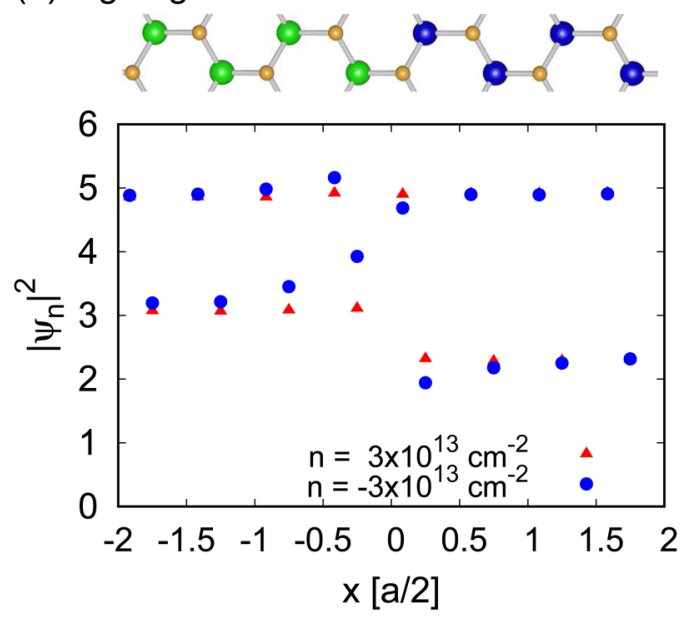

(b) Armchair interface

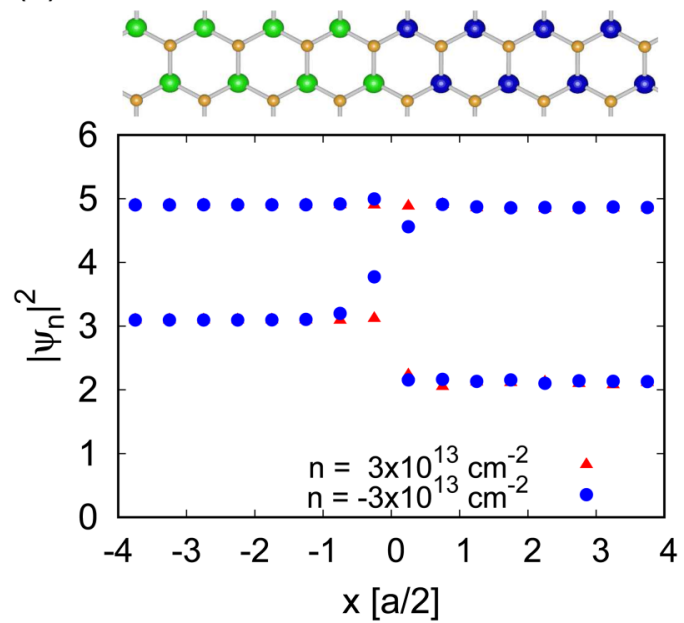

FIG. 5. The charge distribution around the interface for $n=-3 \times 10^{13} \mathrm{~cm}^{-2}$ and $n=3 \times 10^{13} \mathrm{~cm}^{-2}$. Here, $x$ indicates the position in the unit of half the unit cell and the interface is placed at $x=0$. The vertical axis indicates the summation of wave amplitude over all the occupied states in the tight-binding model. The schematic of the lattice structure represents the atomic positions.

a periodic boundary condition with 20 unit cells aligned in the $x$ axis. The vertical axis indicates the wave amplitude of adopted orbitals, which is included in the tight-binding model, for the occupied states, and the horizontal axis exhibits the position in the $x$ axis with the interface at $x=0$. The wave amplitude is nearly $\left|\psi_{n}\right|^{2}=5$ at Se atoms in both the regions, and in the transitionmetal atoms, the wave amplitude changes between two regions due to the difference of atomic numbers for $\mathrm{Mo}$ and $\mathrm{Nb}$. Around the interface $x=0$, the charge distribution varies from that far from the interface $2 \leq|x|$. In the electron-doped junction (circle symbols in Fig. 5), the charge transfer arises between two regions, and it induces the drastic fluctuation of the charge density at the vicinity of the interface. In the hole-doped junction (triangle symbols in Fig. 5), on the other hand, the charge distribution slightly fluctuates even in the vicinity of the interface. The difference between the electron-doped and hole-doped cases is discussed in Sec. V.

We calculate the transmission probability $\tilde{t}_{\mu v}\left(k_{y}\right)$ by using the lattice Green's function method for the multiorbital tight-binding model, ${ }^{8,43,44}$ where $v$ and $\mu$ represent the incoming and outgoing waves in $\mathrm{MoSe}_{2}$ and $\mathrm{NbSe}_{2}$, respectively. At the zero temperature, the conductance per unit width is given by

$$
g_{e}\left(E_{F}\right)=\frac{e^{2}}{\pi \hbar} \int \frac{d k_{y}}{2 \pi} \sum_{\mu, v}\left|\sqrt{\frac{v_{\mu}}{v_{v}}} \tilde{t}_{\mu v}\left(k_{y}\right)\right|^{2} .
$$

Here, $v_{\mu}$ and $v_{v}$ are the velocity along the $x$ axis for the outgoing wave in $\mathrm{NbSe}_{2}$ and the incoming wave in $\mathrm{MoSe}_{2}$, respectively. The $\mathrm{NbSe}_{2}$ monolayer shows the phase transition, CDW and superconductivity, at low temperature, but we consider normal states of $\mathrm{NbSe}_{2}$ for investigating the fundamental transport property of the metallic $\mathrm{NbSe}_{2}$ monolayer. We define the positive direction in the $x$ axis as the transmission direction. Thus, the electronic waves $\mu$ and $v$ have the positive velocity in the $x$ axis. The wave functions are represented by the periodic part $\boldsymbol{c}_{j}$, wave amplitudes of Wannier functions, in a unit cell and the phase shift $\lambda=e^{-i \phi}$ in the transmission process between adjacent cells. The coefficients are the eigenfunction and eigenvalue of the following equation for each $k_{y}$ in pristine $\mathrm{MoSe}_{2}$ and $\mathrm{NbSe}_{2}$ monolayers as

$$
\lambda\left(\begin{array}{c}
\boldsymbol{c}_{j} \\
\boldsymbol{c}_{j-1}
\end{array}\right)=\left(\begin{array}{cc}
\hat{h}_{1}^{-1}\left(k_{y}\right)\left(\hat{h}_{0}\left(k_{y}\right)-E_{F}\right) & -\hat{h}_{1}^{-1}\left(k_{y}\right) \hat{h}_{-1}\left(k_{y}\right) \\
1 & 0
\end{array}\right)\left(\begin{array}{c}
\boldsymbol{c}_{j} \\
\boldsymbol{c}_{j-1}
\end{array}\right),
$$

with the on-site potential $\hat{h}_{0}\left(k_{y}\right)$ and the right-going and left-going hopping matrix $\hat{h}_{1}$ and $\hat{h}_{-1}$, respectively. The velocity in the $x$ axis is calculated by $v_{x}=\left(\boldsymbol{c}_{j}, \boldsymbol{c}_{j-1}\right)^{\dagger} \hat{v}_{x}\left(\boldsymbol{c}_{j}, \boldsymbol{c}_{j-1}\right)$ with the velocity operator defined by

$$
\hat{v}_{x}=-i \frac{a_{x}}{\hbar}\left(\begin{array}{cc}
0 & \hat{h}_{-1}\left(k_{y}\right) \\
\hat{h}_{1}\left(k_{y}\right) & 0
\end{array}\right),
$$

where $a_{x}$ is the length of the square unit cell in Fig. 1 along $x$. The relation between the Fermi energy and the charge density is represented in Eq. (1). We consider the charge induction by using a top or back gate being homogeneous in the $x y$ plane. By gating, opposite charges are induced in the substrate and homogeneously distributed in the $x y$ plane. Thus, we can assume that the induced charges in the heterojunction are distributed homogeneously, i.e., $n=$ const, in the $x y$ plane due to the local charge neutrality. Then, the Fermi energy is also applied to the $\mathrm{MoSe}_{2}$ and $\mathrm{NbSe}_{2}$ regions in the lateral heterojunction. In a realistic interface, the charge distribution is more complicated, but we adopt the approximation for analyzing an ideal transmission case and for simplicity. We also calculate $k_{x}$ of incoming and outgoing waves by using $\phi=k_{x} c_{x}$, and thus, we can characterize these waves by the valley degree of freedom $\tau$ by referring to the Fermi surface in Figs. 3 and 4. Therefore, the wave index, $\mu$ and $v$, is given by the valley index $\tau$ and the spin index $s$. By picking up a spin 
and a valley, it is possible to calculate the conductance of spinpolarized electrons transferring between two valleys.

We also consider the conductance at nonzero temperatures. The temperature does not change the transmission probability at the interface because the width of the scattering region, the interface, is always shorter than the coherent length. The incident electrons, on the other hand, are distributed in the energy around the Fermi level due to the temperature $T$ and described by the Fermi distribution function $f_{F}(E, T)=\left(1+\exp \left[\left(E-E_{F}\right) / k_{B} T\right]\right)^{-1}$ with the Boltzmann constant $k_{B}$. At nonzero temperature, the conductance can be calculated by using the Landauer formula at the finite temperature,

$$
g_{e}\left(E_{F}, T\right)=\int_{-\infty}^{\infty} d E\left(-\frac{\partial f_{F}(E, T)}{\partial E}\right) g_{e}(E)
$$

Here, the derivative of the Fermi distribution function becomes the Dirac delta function $\delta\left(E-E_{F}\right)$ at zero temperature, and thus, the formula reproduces the conductance in Eq. (2) at the zero temperature. In Fig. 6, we show the conductance at $50 \mathrm{~K}$ and $150 \mathrm{~K}$, where both the temperatures are above the phase transition temperature to $\mathrm{CDW} T_{\mathrm{CDW}}=33 \mathrm{~K}-45 \mathrm{~K}$ on the bilayer graphene substrate ${ }^{38}$ and $145 \mathrm{~K}$ on the sapphire substrate. ${ }^{39}$

(a) Armchair-type interface

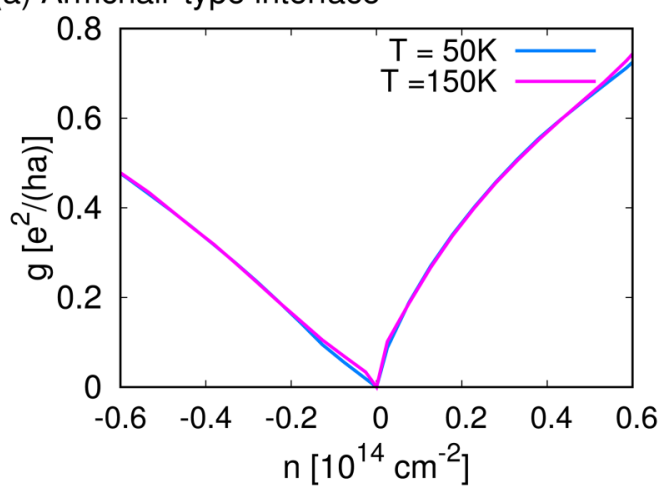

(b) Zig-zag-type interface

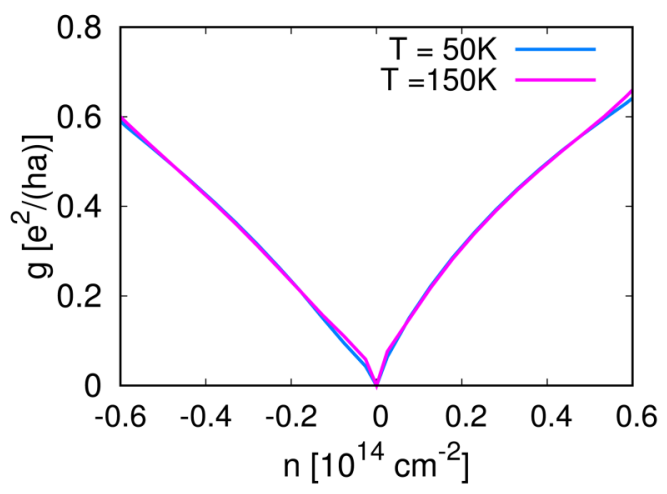

FIG. 6. Temperature dependence of electric conductance in the lateral heterostructure with the armchair interface (a) and the zigzag interface (b).

\section{NUMERICAL RESULTS}

First, we show the charge density-dependence of electric conductance $g_{e}$ in Fig. 7. The conductance drops around $n=0$ in both the two types of heterostructures with an armchair-type interface and a zigzag-type interface in Figs. 1(a) and 1(b), respectively, and it monotonically increases with $|n|$. This is because the Fermi level is in the insulating gap under $n=0$, and the conducting channels increase with $|n|$ in $\mathrm{MoSe}_{2}$. At every nonzero charge densities, the heterostructure with the armchair-type interface gives a larger $g_{e}$ than that with the zigzag-type interface in the hole-doped junctions. The armchair interface allows electrons transferring between different valleys, e.g., $K$ and $\Gamma$, in $\mathrm{MoSe}_{2}$ and $\mathrm{NbSe}_{2}$ as discussed below, and thus, it enhances the total transmission probability. In the transmission process, the electronic spin is conserved due to the mirror symmetry in the $z$ axis, the out-of-plane direction. Thus, the conductance $g_{e}$ is separated into that of up-spin electrons $g_{\uparrow}$ and down-spin electrons $g_{\downarrow}$. In TMDC monolayers, the spin relaxation is suppressed even in the presence of the nonmagnetic impurity due to the mirror symmetry. ${ }^{44-46}$ The two spin components are

(a) Armchair-type interface

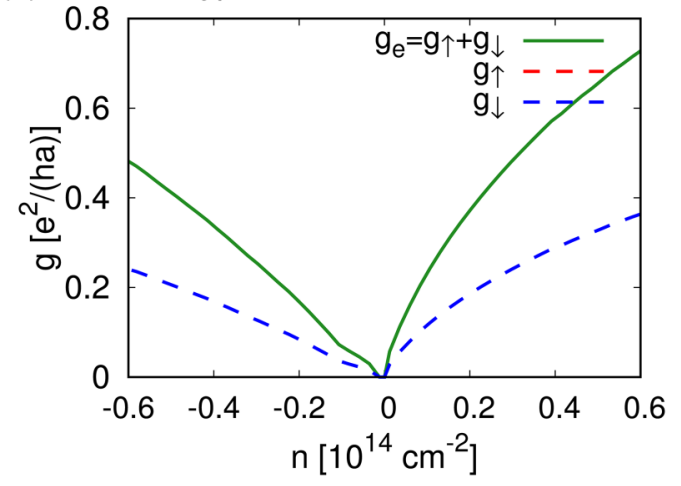

(b) Zig-zag-type interface

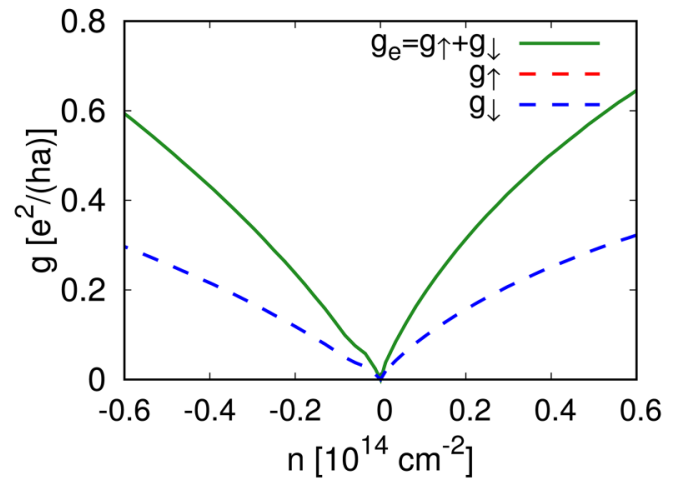

FIG. 7. The charge density-dependence of electric conductance per unit width (solid line) in the heterojunction with an armchair-type interface (a) and a zigzag-type interface (b). The dashed lines indicate the conductivity for each spin state. 
(a) $\mathrm{K}\left(\mathrm{MoSe}_{2}\right)-\mathrm{K}\left(\mathrm{NbSe}_{2}\right)$

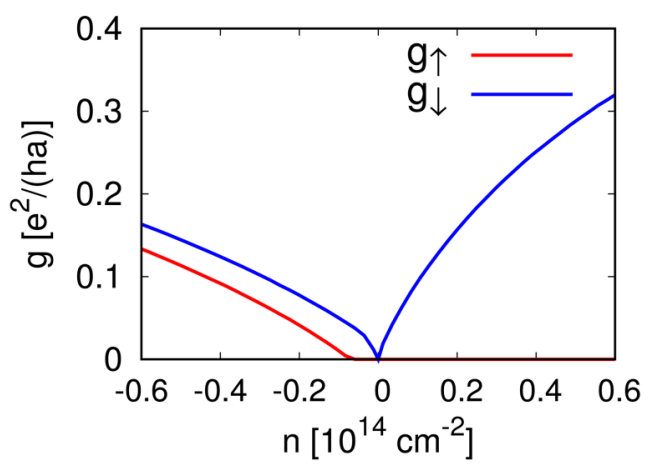

(b) $\mathrm{K}^{\prime}\left(\mathrm{MoSe}_{2}\right)-\mathrm{K}^{\prime}\left(\mathrm{NbSe}_{2}\right)$

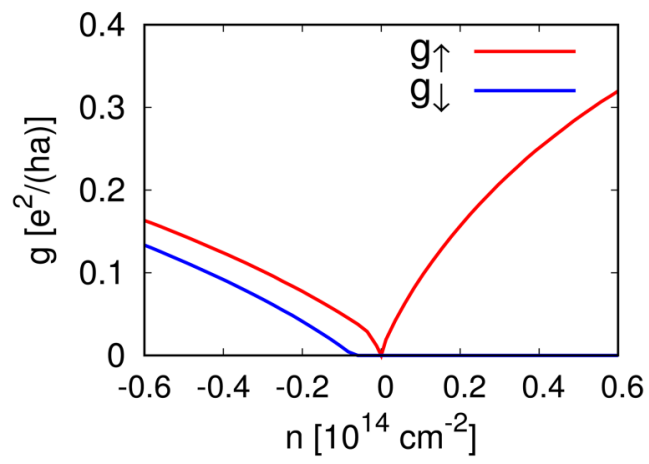

FIG. 8. Valley and spin dependence of electric conductance in the lateral heterostructure with a zigzag interface. (a) and (b) represent the transmission of the electron in the $K$ and $K^{\prime}$ valleys, respectively.

balanced due to the equal population of two spins in the presence of the time-reversal symmetry.

Next, we show the valley-dependent conductance in the two types of heterostructures in Figs. 8 and 9. The heterostructure with a zigzag interface preserves the mirror symmetry in the $y$ axis. Thus, the transmission probability is equivalent in both the valleys as shown in Fig. 8. When passing through the zigzag interface, electrons preserve the valley degree of freedom due to conservation of wave number $k_{y}$ parallel to the interface. Here, $k_{y}$ is parallel to the $M-K$ direction in Figs. 3 and 4 , and the transmission of the electron with $k_{y}$ is nonzero as long as electronic states are present in the Fermi surface of both $\mathrm{MoSe}_{2}$ and $\mathrm{NbSe}_{2}$. Since the spin is fully polarized in the $K$ and $K^{\prime}$ valleys on the condition of $0<n$ in $\mathrm{MoSe}_{2}$, the spin and valley are locked to each other in the transmission process. In $n<0$, on the other hand, the up-spin and down-spin electrons flow above $n=3 \times 10^{13} \mathrm{~cm}^{-2}$ in both the two valleys.

In Fig. 9, we show the spin and valley dependence of conductance of the heterojunction with an armchair-type interface. Valley-conserving transmission probability is similar to that in the zigzag interface. Moreover, electrons in $\mathrm{MoSe}_{2}$ are able to transmit with switching the valley to $\Gamma$ as shown in (b) and (e), but the valley-switch between the $K$ and $K^{\prime}$ valleys is highly suppressed even in this heterostructure as shown in (c) and (d). The electronic flow in the $\Gamma$ valley carries the spin current in $0<n$. The spin flow is attributed to the difference of the transmission coefficient for incoming electronic waves in the $K$ and $K^{\prime}$ valleys of $\mathrm{MoSe}_{2}$. Here, the transmission coefficient is defined ast $t_{\mu v}=\sqrt{v_{\mu} / v_{v}} \tilde{t}_{\mu v}$, which is the integrand in Eq. (2), and it depends on the ratio of the Fermi velocity $v_{u} / v_{v}$ in the pristine $\mathrm{NbSe}_{2}$ and $\mathrm{MoSe}_{2}$. We show the Fermi velocity in $\mathrm{MoSe}_{2}$ and $\mathrm{NbSe}_{2}$ in Fig. 10, where the electronic states having the right-going velocity and being inside of dashed lines correspond to the wave vector holding conducting channels. The Fermi velocity of right-going states with down-spin is much smaller than that with up-spin in $\mathrm{NbSe}_{2}$. The spin current in the $\Gamma$ valley is caused by this anisotropy of the Fermi velocity. The armchair interface allows us to obtain not only the spin-polarized electronic current in the $\Gamma$ valleys but also the valley polarized current between the $K$ and $K^{\prime}$ valleys, which can be observed by the valley Hall effect due to the opposite Berry curvature in the two valleys. ${ }^{1,3,47}$

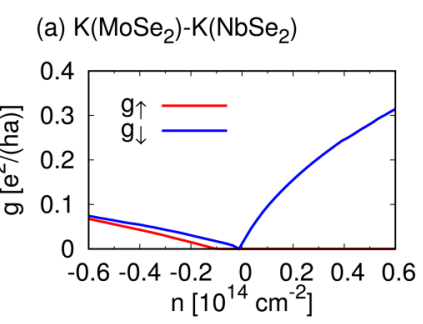

(d) $\mathrm{K}^{\prime}\left(\mathrm{MoSe}_{2}\right)-\mathrm{K}\left(\mathrm{NbSe}_{2}\right)$

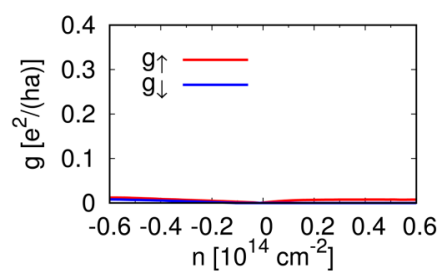

(b) $\mathrm{K}\left(\mathrm{MoSe}_{2}\right)-\Gamma\left(\mathrm{NbSe}_{2}\right)$

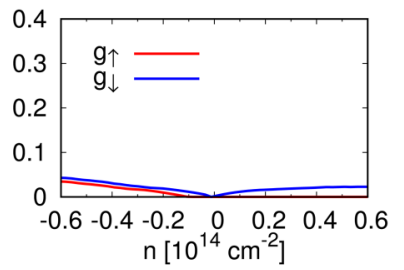

(e) $\mathrm{K}^{\prime}\left(\mathrm{MoSe}_{2}\right)-\Gamma\left(\mathrm{NbSe}_{2}\right)$

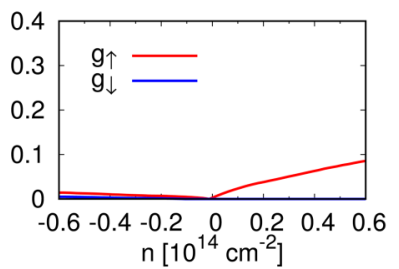

(c) $\mathrm{K}\left(\mathrm{MoSe}_{2}\right)-\mathrm{K}^{\prime}\left(\mathrm{NbSe}_{2}\right)$

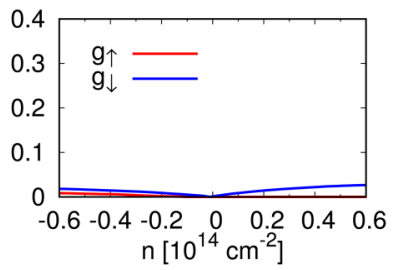

(f) $\mathrm{K}^{\prime}\left(\mathrm{MoSe}_{2}\right)-\mathrm{K}^{\prime}\left(\mathrm{NbSe}_{2}\right)$

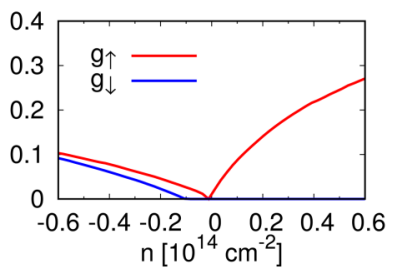

FIG. 9. Valley and spin dependence of electric conductance in the lateral heterostructure with an armchair interface. Upper panels (a)-(c) and lower panels (d)-(f) represent the transmission of the electron in the $K$ and $K^{\prime}$ valleys of $\mathrm{MoSe}_{2}$. 
Monolayer $\mathrm{MoSe}_{2}$

(a) Up-spin (K')

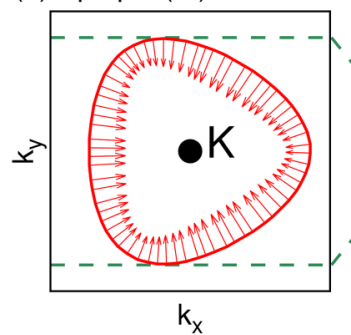

(c) Down-spin (K)
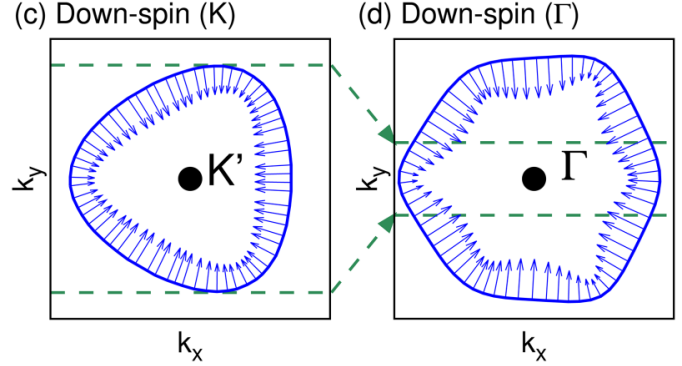

FIG. 10. Fermi velocity in $\mathrm{MoSe}_{2}$ and $\mathrm{NbSe}_{2}$ with $n=3 \times 10^{13} \mathrm{~cm}^{-2}$. Dashed lines indicate the upper and lower $k_{y}$ at which conducting channels are present in $\mathrm{MoSe}_{2}$. The arrow represents the Fermi velocity, and its length is the relative speed.

\section{DISCUSSION}

In Sec. IV, we consider the heterojunction with a charge density induced by electrostatic gating. In this section, we discuss the effect of chemical doping in the lateral heterojunction. The dopants form impurity levels inside the gap of the semiconductor and pin the Fermi energy at a level without gating. Around the interface, the charge density is modulated due to the difference of the work function between $\mathrm{MoSe}_{2}$ and $\mathrm{NbSe}_{2}$. We calculate the electrostatic potential for electrons as a function of $z$, the out-of-plane coordinate, by using quantum-ESPRESSO and plot it referring to the Fermi level in Fig. 11. Here, we set the origin of $z$ at the transition-metal atoms and adopt the potential at $z / d_{\mathrm{Se}-\mathrm{Se}}=2$ as the vacuum level. The work function is defined by the vacuum level with respect to the Fermi energy. We compute the work function $W_{\mathrm{MoSe}_{2}}=5.217 \mathrm{eV}$ and $W_{\mathrm{NbSe}_{2}}=5.447 \mathrm{eV}$, where $W_{\mathrm{MoSe}_{2}}$ is similar to a previous work ${ }^{48}$ and obtain the difference $W_{\mathrm{MoSe}_{2}}-W_{\mathrm{NbSe}_{2}}=-0.230 \mathrm{eV}$. Since the semiconducting TMDC, $\mathrm{MoSe}_{2}$, has a smaller work function than the metallic TMDC, $\mathrm{NbSe}_{2}$, a Schottky barrier is formed in the junction of electron-doped $\mathrm{MoSe}_{2}$ due to the modulation of the doped carrier density. In the hole-doped heterostructure, on the other hand, the junction has a Ohmic contact.

Around the interface of realistic materials, charges transfer between two TMDCs due to the difference of work functions as shown in Fig. 5. The charge transfer modifies the Fermi energy near the interface, and it can be treated as a contact resistance. It reduces the transmission probability, but the spin and valley polarization is unchanged qualitatively because time-reversal and mirror symmetries are preserved. Furthermore, the control of contact

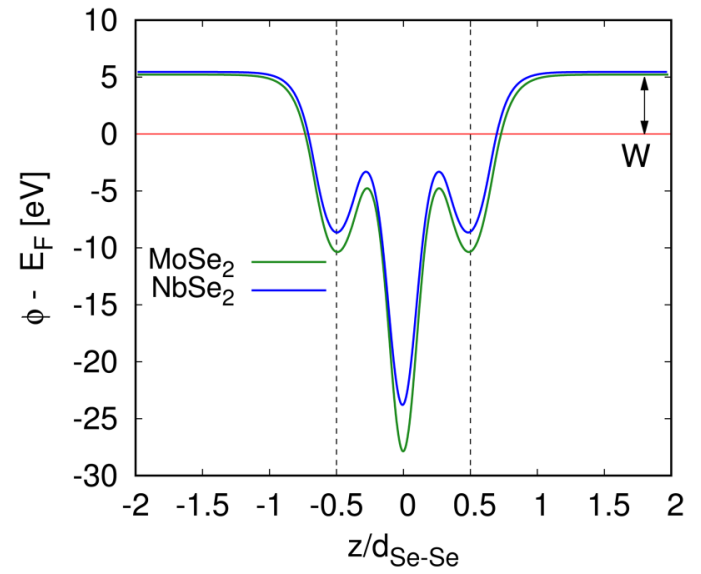

FIG. 11. Spatially electrostatic potential as a function of the out-of-plane coordinate in pristine $\mathrm{MoSe}_{2}$ and $\mathrm{NbSe}_{2}$ monolayers. The dashed lines indicate the positions of chalcogen atoms.

resistance for atomic layered materials has been developing, e.g., the graphene-metal junction. ${ }^{49}$ In our calculation, we assume the uniform Fermi energy over each TMDC for analyzing an ideal transmission case.

Finally, we discuss the experimental observation associated with the spin and valley-dependent transmission in the lateral heterojunction. We show that the spin of conduction electrons is not
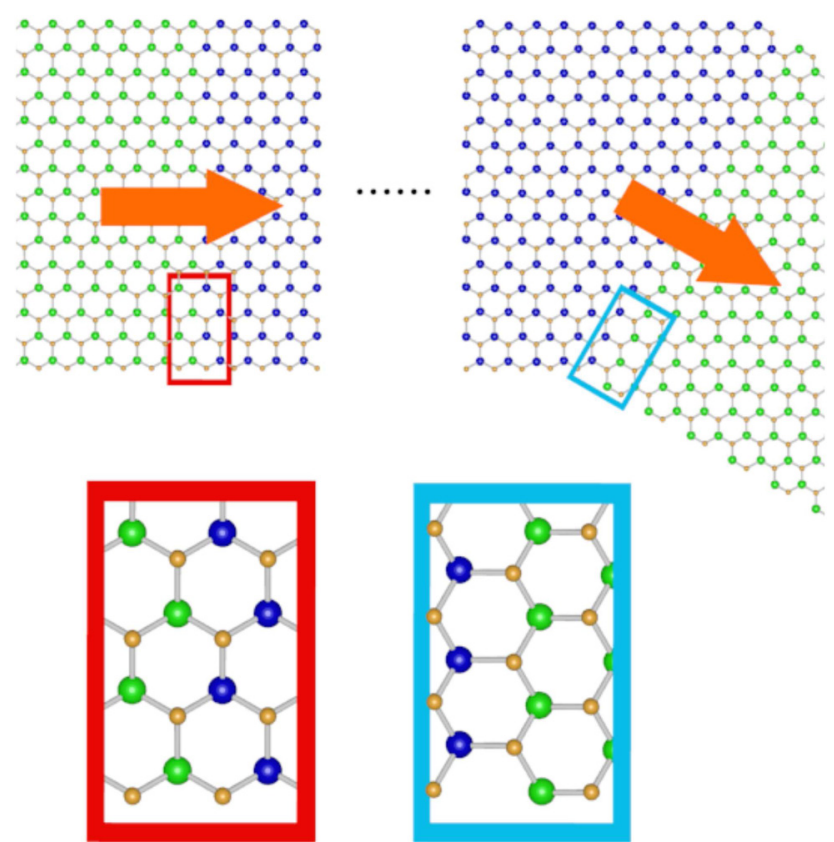

FIG. 12. The schematic of MoSe2-NbSe $-\mathrm{MoSe}_{2}$ junction with two boundaries The arrows indicate the current direction. 
polarized at a single heterojunction as shown in Fig. 7. By combining the armchair and zigzag interfaces, on the other hand, the two-interface heterojunction induces the spin and valley polarized current. In Fig. 12, the left interface changes the transmission probability between $K$ and $K^{\prime}$, and the right interface allows the equal transmission for the two valleys. Then, the spin and valley polarization induced by the left interface remains in the right $\mathrm{MoSe}_{2}$ region. Therefore, the spin- and valley-dependent transmission can be shown in the two-interface heterojunction experimentally.

\section{CONCLUSION}

We have investigated the electronic transport property of lateral heterojunctions of the metallic $\mathrm{NbSe}_{2}$ monolayer and the semiconducting $\mathrm{MoSe}_{2}$ monolayer and have shown that the spin and valley dependence of conductance changes with the interface structure. The zigzag-type interface conserves the spin and valley of conduction electrons in the $K$ and $K^{\prime}$ valleys, and the conductance is independent of the valley and spin. This means that $\mathrm{NbSe}_{2}$ can be applied to the lead in spintronic or valleytronic devices of semiconducting TMDCs. When electrons pass through an armchair-type interface, the electronic spin is also conserved, but the valley, on the other hand, changes in the transmission process. This valley-flip increases the total transmission probability, and thus, the conductance is larger than that with the zigzag-type interface in $0<n$. In the case of $n<0$, the probability of the valley-conserving transmission is suppressed in the case of the armchair-type interface as shown in Figs. 8 and 9. Thus, the conductance is enhanced in the junction with the zigzag-type interface. Moreover, the transmission probability is strongly dependent on the spin and valley, and thus, conduction electrons are spin-polarized in each valley. The lateral heterostructure can be useful in spintronics and valleytronics, and its transport properties studied here provide the opportunity to develop devices made by only atomic layers.

\section{REFERENCES}

${ }^{1}$ D. Xiao, G.-B. Liu, W. Feng, X. Xu, and W. Yao, Phys. Rev. Lett. 108, 196802 (2012).

${ }^{\mathbf{2}}$ N. Zibouche, A. Kuc, J. Musfeldt, and T. Heine, Ann. Phys. 526, 395 (2014).

${ }^{3}$ W.-Y. Shan, H.-Z. Lu, and D. Xiao, Phys. Rev. B 88, 125301 (2013).

${ }^{4}$ J. Klinovaja and D. Loss, Phys. Rev. B 88, 075404 (2013).

${ }^{\mathbf{5}}$ K. F. Mak, K. He, J. Shan, and T. F. Heinz, Nat. Nanotechnol. 7, 494 (2012).

${ }^{6}$ R. Suzuki, M. Sakano, Y. J. Zhang, R. Akashi, D. Morikawa, A. Harasawa, K. Yaji, K. Kuroda, K. Miyamoto, T. Okuda, K. Ishizaka, R. Arita, and Y. Iwasa, Nat. Nanotechnol. 9, 611 (2014).

${ }^{7}$ H. Yuan, X. Wang, B. Lian, H. Zhang, X. Fang, B. Shen, G. Xu, Y. Xu, S.-C. Zhang, H. Y. Hwang, and Y. Cui, Nat. Nanotechnol. 9, 851 (2014).

${ }^{8}$ T. Habe and M. Koshino, Phys. Rev. B 91, 201407 (2015).

${ }^{9}$ Q. Shao, G. Yu, Y.-W. Lan, Y. Shi, M.-Y. Li, C. Zheng, X. Zhu, L.-J. Li, P. K. Amiri, and K. L. Wang, Nano Lett. 16, 7514 (2016).

${ }^{10}$ T. Cao, G. Wang, W. Han, H. Ye, C. Zhu, J. Shi, Q. Niu, P. Tan, E. Wang, B. Liu, and J. Feng, Nature Commun. 3, 887 (2012).

${ }^{11}$ H. Zeng, J. Dai, W. Yao, D. Xiao, and X. Cui, Nat. Nanotechnol. 7, 490 (2012).

${ }^{12}$ Z. Gong, G.-B. Liu, H. Yu, D. Xiao, X. Cui, X. Xu, and W. Yao, Nat. Commun. 4, 2053 (2013).

${ }^{13}$ Y. Ye, J. Xiao, H. Wang, Z. Ye, H. Zhu, M. Zhao, Y. Wang, J. Zhao, X. Yin, and X. Zhang, Nat. Nanotechnol. 11, 598 (2016).
${ }^{14}$ S. Helveg, J. V. Lauritsen, E. Lægsgaard, I. Stensgaard, J. K. Nørskov, B. S. Clausen, H. Topsøe, and F. Besenbacher, Phys. Rev. Lett. 84, 951 (2000).

${ }^{15}$ K. F. Mak, C. Lee, J. Hone, J. Shan, and T. F. Heinz, Phys. Rev. Lett. 105, 136805 (2010).

${ }^{16}$ J. N. Coleman, M. Lotya, A. O’Neill, S. D. Bergin, P. J. King, U. Khan, K. Young, A. Gaucher, S. De, R. J. Smith, I. V. Shvets, S. K. Arora, G. Stanton, H.-Y. Kim, K. Lee, G. T. Kim, G. S. Duesberg, T. Hallam, J. J. Boland, J. J. Wang, J. F. Donegan, J. C. Grunlan, G. Moriarty, A. Shmeliov, R. J. Nicholls, J. M. Perkins, E. M. Grieveson, K. Theuwissen, D. W. McComb, P. D. Nellist, and V. Nicolosi, Science 331, 568 (2011).

${ }^{17}$ Y.-H. Lee, X.-Q. Zhang, W. Zhang, M.-T. Chang, C.-T. Lin, K.-D. Chang, Y.-C. Yu, J. T.-W. Wang, C.-S. Chang, L.-J. Li, and T.-W. Lin, Adv. Mater. 24, 2320 (2012).

${ }^{18}$ R. Dong and I. Kuljanishvili, J. Vac. Sci. Technol. B 35, 030803 (2017).

${ }^{19}$ J. He, K. Hummer, and C. Franchini, Phys. Rev. B 89, 075409 (2014).

${ }^{20}$ Z. Ji, H. Hong, J. Zhang, Q. Zhang, W. Huang, T. Cao, R. Qiao, C. Liu, J. Liang, C. Jin, L. Jiao, K. Shi, S. Meng, and K. Liu, ACS Nano 11, 12020 (2017).

${ }^{21}$ M. Baranowski, A. Surrente, L. Klopotowski, J. M. Urban, N. Zhang, D. K. Maude, K. Wiwatowski, S. Mackowski, Y. C. Kung, D. Dumcenco, A. Kis, and P. Plochocka, Nano Lett. 17, 6360 (2017).

${ }^{22}$ W. Xu, D. Kozawa, Y. Liu, Y. Sheng, K. Wei, V. B. Koman, S. Wang, X. Wang, T. Jiang, M. S. Strano, and J. H. Warner, Small 14, 1703727 (2018).

${ }^{23}$ C. Huang, S. Wu, A. M. Sanchez, J. J. P. Peters, R. Beanland, J. S. Ross, P. Rivera, W. Yao, D. H. Cobden, and X. Xu, Nat. Mater. 13, 1096 (2014).

${ }^{24}$ Y. Gong, J. Lin, X. Wang, G. Shi, S. Lei, Z. Lin, X. Zou, G. Ye, R. Vajtai, B. I. Yakobson, H. Terrones, M. Terrones, B. Tay, J. Lou, S. T. Pantelides, Z. Liu, W. Zhou, and P. M. Ajayan, Nat. Mater. 13, 1135 (2014).

${ }^{25}$ X. Duan, C. Wang, J. C. Shaw, R. Cheng, Y. Chen, H. Li, X. Wu, Y. Tang, Q. Zhang, A. Pan, J. Jiang, R. Yu, Y. Huang, and X. Duan, Nat. Nanotechnol. 9, 1024 (2014).

${ }^{26}$ K. Chen, X. Wan, W. Xie, J. Wen, Z. Kang, X. Zeng, H. Chen, and J. Xu, Adv. Mater. 27, 6431 (2015).

${ }^{27}$ K. Chen, X. Wan, J. Wen, W. Xie, Z. Kang, X. Zeng, H. Chen, and J.-B. Xu, ACS Nano 9, 9868 (2015).

${ }^{28}$ X.-Q. Zhang, C.-H. Lin, Y.-W. Tseng, K.-H. Huang, and Y.-H. Lee, Nano Lett. 15, 410 (2015).

${ }^{29}$ Y. He, A. Sobhani, S. Lei, Z. Zhang, Y. Gong, Z. Jin, W. Zhou, Y. Yang, Y. Zhang, X. Wang, B. Yakobson, R. Vajtai, N. J. Halas, B. Li, E. Xie, and P. Ajayan, Adv. Mater. 28, 5126 (2016).

${ }^{30}$ J. Kang, S. Tongay, J. Zhou, J. Li, and J. Wu, Appl. Phys. Lett. 102, 012111 (2013).

${ }^{31}$ C. Zhang, Y. Nie, T. Liao, L. Kou, and A. Du, Phys. Rev. B 99, 035424 (2019).

${ }^{32}$ M.-Y. Li, Y. Shi, C.-C. Cheng, L.-S. Lu, Y.-C. Lin, H.-L. Tang, M.-L. Tsai, C.-W. Chu, K.-H. Wei, J.-H. He, W.-H. Chang, K. Suenaga, and L.-J. Li, Science 349, 524 (2015).

${ }^{33}$ M. Najmzadeh, C. Ko, K. Wu, S. Tongay, and J. Wu, Appl. Phys. Express 9, 055201 (2016).

${ }^{34}$ F. Ullah, Y. Sim, C. T. Le, M.-J. Seong, J. I. Jang, S. H. Rhim, B. C. Tran Khac, K.-H. Chung, K. Park, Y. Lee, K. Kim, H. Y. Jeong, and Y. S. Kim, ACS Nano 11, 8822 (2017).

${ }^{35}$ Y. Son, M.-Y. Li, C.-C. Cheng, K.-H. Wei, P. Liu, Q. H. Wang, L.-J. Li, and M. S. Strano, Nano Lett. 16, 3571 (2016).

${ }^{36}$ J. M. Lu, O. Zheliuk, I. Leermakers, N. F. Q. Yuan, U. Zeitler, K. T. Law, and J. T. Ye, Science 350, 1353 (2015).

${ }^{37}$ H. Wang, X. Huang, J. Lin, J. Cui, Y. Chen, C. Zhu, F. Liu, Q. Zeng, J. Zhou, P. Yu, X. Wang, H. He, S. H. Tsang, W. Gao, K. Suenaga, F. Ma, C. Yang, L. Lu, T. Yu, E. H. T. Teo, G. Liu, and Z. Liu, Nat. Commun. 8, 394 (2017).

${ }^{38}$ M. M. Ugeda, A. J. Bradley, Y. Zhang, S. Onishi, Y. Chen, W. Ruan, C. Ojeda-Aristizabal, H. Ryu, M. T. Edmonds, H.-Z. Tsai, A. Riss, S.-K. Mo, D. Lee, A. Zettl, Z. Hussain, Z.-X. Shen, and M. F. Crommie, Nat. Phys. 12, 92 (2015).

${ }^{39} \mathrm{X}$. Xi, L. Zhao, Z. Wang, H. Berger, L. Forró, J. Shan, and K. F. Mak, Nat. Nanotechnol. 10, 765 (2015). 
${ }^{40}$ P. Giannozzi, S. Baroni, N. Bonini, M. Calandra, R. Car, C. Cavazzoni, D. Ceresoli, G. L. Chiarotti, M. Cococcioni, I. Dabo, A. Dal Corso, S. de Gironcoli, S. Fabris, G. Fratesi, R. Gebauer, U. Gerstmann, C. Gougoussis, A. Kokalj, M. Lazzeri, L. Martin-Samos, N. Marzari, F. Mauri, R. Mazzarello, S. Paolini, A. Pasquarello, L. Paulatto, C. Sbraccia, S. Scandolo, G. Sclauzero, A. P. Seitsonen, A. Smogunov, P. Umari, and R. M. Wentzcovitch, J. Phys. Condens. Matter 21, 395502 (2009).

${ }^{41}$ Y. Zhang, J. Ye, Y. Matsuhashi, and Y. Iwasa, Nano Lett. 12, 1136 (2012).

${ }^{42}$ A. A. Mostofi, J. R. Yates, Y.-S. Lee, I. Souza, D. Vanderbilt, and N. Marzari, Comput. Phys. Commun. 178, 685 (2008).
${ }^{43}$ T. Ando, Phys. Rev. B 44, 8017 (1991).

${ }^{44}$ T. Habe and M. Koshino, Phys. Rev. B 93, 075415 (2016).

${ }^{45} \mathrm{H}$. Ochoa and R. Roldán, Phys. Rev. B 87, 245421 (2013).

${ }^{46} \mathrm{H}$. Ochoa, F. Guinea, and V. I. Fal'ko, Phys. Rev. B 88, 195417 (2013).

${ }^{47}$ T. Habe and M. Koshino, Phys. Rev. B 96, 085411 (2017).

${ }^{48}$ N. A. Lanzillo, A. J. Simbeck, and S. K. Nayak, J. Phys. Condens. Matter 27, 175501 (2015).

${ }^{49} \mathrm{~F}$. Giubileo and A. D. Bartolomeo, Prog. Surf. Sci. 92, 143 (2017). 